1 Hacettepe Journal of Mathematics and Statistics

$\bigcap$ Volume 47 (3) (2018), 637-648

\title{
A Two-by-Two matrix representation of a generalized Fibonacci sequence
}

Arfat Ahmad Wani * G. P. S. Rathore $\dagger^{\dagger}$ V. H. Badshah $\dagger^{\ddagger}$ and Kiran Sisodiya

\begin{abstract}
The Fibonacci sequence is a well-known example of second order recurrence sequence, which belongs to a particular class of recursive sequences. In this article, other generalized Fibonacci sequence is introduced and defined by $H_{k, n+1}=2 H_{k, n}+k H_{k, n-1}, n \geq 1, H_{k, 0}=$ $2, H_{k, 1}=1$ and $k$ is the positive real number. Also $n^{t h}$ power of the generating matrix for this generalized Fibonacci sequence is established and some basic properties of this sequence are obtained by matrix methods.
\end{abstract}

Keywords: Fibonacci Sequence, Generalized Fibonacci Sequences and Matrix Methods.

2000 AMS Classification: 11B39, 40C05.

Received : 06.12.2016 Accepted : 13.05.2017 Doi : 10.15672/HJMS.2017.477

\section{Introduction}

The well-known Fibonacci sequence is a sequence of positive integers that has been studied over several years. Many authors are dedicated to study this sequence. The most and vast research field of Fibonacci numbers are dedicated to study the generalizations of Fibonacci numbers [6, 11]. The main aim of the present paper is to study other generalized Fibonacci sequence by matrix methods.

Horadam [3] introduced and studied the generalized Fibonacci sequence $W_{n}=W_{n}(a, b ; p, q)$ defined by

$$
W_{n}=p W_{n-1}-q W_{n-2}, n \geq 1, W_{0}=a, W_{1}=b
$$

*School of Studies in Mathematics, Vikram University Ujjain, Ujjain, India.

Email : arfatmaths@gmail.com

$\dagger$ Department of Statistics, Horticulture College Mandsaur, Mandsaur, India.

Email : gps_rathore20@yahoo.co.in

${ }^{\ddagger}$ School of Studies in Mathematics, Vikram University Ujjain, India.

Email : vhbadshah@gmail.com

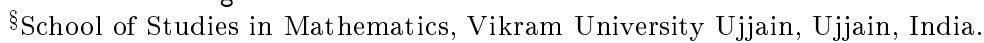

Email : sisodiya.kiran4@gmail.com 
where $a, b, p$ and $q$ are arbitrary complex numbers with $q \neq 0$. These numbers were first studied by Horadam and they are called Horadam numbers. In [7] Silvester shows that a number of the properties of the Fibonacci sequence can be derived from a matrix representation. In doing so, he showed that if

$$
A=\left[\begin{array}{ll}
0 & 1 \\
1 & 1
\end{array}\right] \text { then } A^{n}\left[\begin{array}{l}
0 \\
1
\end{array}\right]=\left[\begin{array}{c}
F_{n} \\
F_{n+1}
\end{array}\right]
$$

where $\left\{F_{n}\right\}$ represents the $n^{\text {th }}$ Fibonacci number. In [5] Koken and Bozkurt obtained some important properties of Jacobsthal numbers by matrix methods, using diagonalization of a $2 \times 2$ matrix to obtain a Binet's formula for the Jacobsthal numbers and in that paper, $2 \times 2$ matrix and its $n^{\text {th }}$ power are defined respectively as

$$
F=\left[\begin{array}{ll}
1 & 2 \\
1 & 0
\end{array}\right] \text { and } F^{n}=\left[\begin{array}{cc}
J_{n+1} & 2 J_{n} \\
J_{n} & 2 J_{n-1}
\end{array}\right]
$$

where $J_{n}$ is the $n^{\text {th }}$ Jacobsthal number. In 4 Demirturk obtained summation formulae for the Fibonacci and Lucas sequences by matrix methods. For doing this, he considered $2 \times 2$ matrix such as

$$
S=\left[\begin{array}{cc}
\frac{1}{2} & \frac{5}{2} \\
\frac{1}{2} & \frac{1}{2}
\end{array}\right] \text { and } S^{n}=\left[\begin{array}{cc}
\frac{L_{n}}{2} & \frac{5 F_{n}}{2} \\
\frac{F_{n}}{2} & \frac{L_{n}}{2}
\end{array}\right]
$$

where $\left\{F_{n}\right\}$ and $\left\{L_{n}\right\}$ are $n^{t h}$ Fibonacci and Lucas numbers respectively. In [10] the authors presented some important relationship between $k$-Jacobsthal matrix sequence and $k$-Jacobsthal-Lucas matrix sequence and $k$ is the positive real number. In [2] Godase and Dhakne described some properties of $k$-Fibonacci and $k$-Lucas numbers by matrix terminology. To obtain such properties, the authors weighed $2 \times 2$ matrix as

$$
S=\left[\begin{array}{cc}
\frac{k}{2} & \frac{k^{2}+4}{2} \\
\frac{1}{2} & \frac{k}{2}
\end{array}\right] \text { and } S^{n}=\left[\begin{array}{cc}
\frac{L_{k, n}}{2} & \frac{\left(k^{2}+4\right) F_{k, n}}{2} \\
\frac{F_{k, n}}{2} & \frac{L_{k, n}}{2}
\end{array}\right]
$$

where $k$ is the fixed positive real number. In 9 Catarino and Vasco introduced a $2 \times 2$ matrix for the $k$-Pell sequence with its $n^{\text {th }}$ power and in [8] Catarino presented Binet's formula for the $k$-Pell sequence by the diagonalization of $2 \times 2$ matrix. In both [8] and [9] the authors defined $2 \times 2$ matrix as

$$
T=\left[\begin{array}{ll}
0 & 1 \\
k & 2
\end{array}\right] \text { and } T^{n}=\left[\begin{array}{cc}
k P_{k, n-1} & P_{k, n} \\
k P_{k, n} & P_{k, n+1}
\end{array}\right]
$$

where $\left\{P_{k, n}\right\}$ is the $n^{t h} k$-Pell number. Again in [8] Catarino obtained Binet's formulae for the $k$-Pell-Lucas sequences by the matrix diagonalization and also obtained some properties of $k$-Pell-Lucas sequence with the help of a $2 \times 2$ matrices defined as

$$
Q=\left[\begin{array}{ll}
0 & 1 \\
k & 2
\end{array}\right] \text { and } Q^{n}=\left[\begin{array}{ll}
k\left(\frac{Q_{k, n}-Q_{k, n-1}}{2(k+1)}\right) & \frac{Q_{k, n+1}-Q_{k, n}}{2(k+1)} \\
k\left(\frac{Q_{k, n+1}-Q_{k, n}}{2(k+1)}\right) & \frac{Q_{k, n+2}-Q_{k, n+1}}{2(k+1)}
\end{array}\right]
$$

where $\left\{Q_{k, n}\right\}$ is the $n^{\text {th }} k$-Pell-Lucas number. In [1] the authors have used the same concept as in [8] and studied $k$-Fibonacci and $k$-Lucas sequences by matrix methods. 


\section{Preliminaries}

In [9] for the positive positive real number $k$ the $k$-Pell sequence $\left\{P_{k, n}\right\}$ is defined by the recurrence relation:

$$
P_{k, n+1}=2 P_{k, n}+k P_{k, n-1}, n \geq 1, P_{k, 0}=0, P_{k, 1}=1
$$

Again in [8] for the positive real number $k$ the $k$-Pell-Lucas sequence $\left\{Q_{k, n}\right\}$ is defined recurrently by

$$
Q_{k, n+1}=2 Q_{k, n}+k Q_{k, n-1}, n \geq 1, Q_{k, 0}=2, Q_{k, 1}=2
$$

The sequences 2.1 and 2.2 have the same characteristic equation $x^{2}-2 x-k=0$ and let $a$ and $b$ are roots of the characteristic equation. The well known general forms of both sequences known as Binet formulae are given and write by

$$
P_{k, n}=\frac{a^{n}-b^{n}}{a-b} \text { and } Q_{k, n}=a^{n}+b^{n}
$$

where $a=1+\sqrt{1+k}$ and $b=1-\sqrt{1+k}$.

The main aim of this paper is to study other generalized Fibonacci sequence by matrix methods, which is defined below:

2.1. Definition. For the positive real number $k$ the generalized Fibonacci sequence, say $\left\{H_{k, n}\right\}$ defined by

$$
H_{k, n+1}=2 H_{k, n}+k H_{k, n-1}, n \geq 1, H_{k, 0}=2, H_{k, 1}=1
$$

Clearly $x^{2}-2 x-k=0$ is also the characteristic equation of the sequence 2.3 . It produces two roots as, $a=1+\sqrt{1+k}$ and $b=1-\sqrt{1+k}$

Some noticeable points about $a$ and $b$ are

$$
a+b=2, \quad a-1=1-b, \quad a^{2}=2 a+k, \quad b^{2}=2 b+k
$$

Also the $2 \times 2$ matrix called generating matrix for the sequence 2.3 is defined as

$$
H=\left[\begin{array}{ll}
2 & k \\
1 & 0
\end{array}\right]
$$

\section{Main Results}

\subsection{Theorem. (Binet Formulae for the Generalized Fibonacci Sequence)}

$$
\begin{aligned}
& H_{k, n}=a^{n}+b^{n}-\frac{a^{n}-b^{n}}{a-b}=Q_{k, n}-P_{k, n}, \quad n \geq 0 \\
& H_{k, n}=\frac{1}{a-b}\left[2\left(a^{n+1}-b^{n+1}\right)-3\left(a^{n}-b^{n}\right)\right]=2 P_{k, n+1}-3 P_{k, n}, \quad n \geq 0
\end{aligned}
$$

Proof. The general form of the generalized Fibonacci sequence may be expressed in the form:

$$
H_{k, n}=A a^{n}+B b^{n}
$$

where $A$ and $B$ are constants that can be determined by the initial conditions. So put the values $n=0$ and $n=1$ in equation $(3.3)$, we get

$$
A+B=2 \text { and } A a+B b=1
$$

After solving the above system of equations for $A$ and $B$, we get

$$
A=\frac{1-2 b}{a-b} \text { and } B=\frac{2 a-1}{a-b}
$$


Therefore,

$$
H_{k, n}=\frac{1}{a-b}\left[a^{n}(1-2 b)-b^{n}(1-2 a)\right]
$$

and by (2.4), we have

$$
\begin{aligned}
H_{k, n} & =\frac{1}{a-b}\left[a^{n}-2 b a^{n}-b^{n}+2 a b^{n}\right] \\
& =\frac{1}{a-b}\left[a^{n}-b a^{n}-b a^{n}-b^{n}+a b^{n}+a b^{n}\right] \\
& =\frac{1}{a-b}\left[a^{n}(1-b)-b^{n}(1-a)-b a^{n}+a b^{n}\right] \\
& =\frac{1}{a-b}\left[a^{n}(a-1)-b^{n}(b-1)-b a^{n}+a b^{n}\right] \\
& =\frac{1}{a-b}\left[a^{n}(a-b)-b^{n}(b-a)-a^{n}+b^{n}\right] \\
& =\frac{1}{a-b}\left[\left(a^{n}+b^{n}\right)(a-b)-\left(a^{n}-b^{n}\right)\right] \\
& =a^{n}+b^{n}-\frac{a^{n}-b^{n}}{a-b}
\end{aligned}
$$

This proves the first part of the theorem (3.1).

Now if we consider equations 2.4 and 3.4 , we get

$$
\begin{aligned}
H_{k, n} & =\frac{1}{a-b}\left[a^{n}-2 b a^{n}-b^{n}+2 a b^{n}\right] \\
& =\frac{1}{a-b}\left[a^{n}-2(2-a) a^{n}+2(2-b) b^{n}-b^{n}\right] \\
& =\frac{1}{a-b}\left[-3 a^{n}+3 b^{n}+2\left(a^{n+1}-b^{n+1}\right)\right] \\
& =\frac{1}{a-b}\left[2\left(a^{n+1}-b^{n+1}\right)-3\left(a^{n}-b^{n}\right)\right]
\end{aligned}
$$

This proves the second part of the theorem 3.1

3.2. Theorem. For $n \in \mathbb{N}$, we have

$$
2 k H_{k, n}-k H_{k, n-1}+2 H_{k, n+2}-H_{k, n+1}=(3+4 k) Q_{k, n}
$$

Proof. To prove this we will use equations $3.1,3.2,2.1$ and $(2.3)$

$$
\begin{aligned}
3 Q_{k, n}+4 k Q_{k, n} & =3\left(H_{k, n}+P_{k, n}\right)+4 k\left(P_{k, n}+H_{k, n}\right) \\
& =3 P_{k, n}+4 k P_{k, n}+3 H_{k, n}+4 k H_{k, n} \\
& =\left(2 P_{k, n+1}-H_{k, n}\right)+4 k P_{k, n}+3 H_{k, n}+4 k H_{k, n} \\
& =2 P_{k, n+1}+4 k P_{k, n}+2 H_{k, n}+4 k H_{k, n} \\
& =4 k P_{k, n}+2 P_{k, n+1}+2 H_{k, n}+4 k H_{k, n} \\
& =4\left(P_{k, n+2}-2 P_{k, n+1}\right)+2 P_{k, n+1}+2 H_{k, n}+4 k H_{k, n} \\
& =4 P_{k, n+2}-6 P_{k, n+1}+2 H_{k, n}+4 k H_{k, n} \\
& =2\left(2 P_{k, n+2}-3 P_{k, n+1}\right)+2 H_{k, n}+4 k H_{k, n} \\
& =2 H_{k, n+1}+2 H_{k, n}+4 k H_{k, n} \\
& =3 H_{k, n+1}-H_{k, n+1}+2 H_{k, n}+4 k H_{k, n} \\
& =3 H_{k, n+1}-k H_{k, n-1}+4 k H_{k, n} \\
& =4 H_{k, n+1}-H_{k, n+1}-k H_{k, n-1}+2 k H_{k, n}+2 k H_{k, n}
\end{aligned}
$$




$$
\begin{aligned}
& =2 k H_{k, n}-k H_{k, n-1}+2\left(2 H_{k, n+1}+k H_{k, n}\right)-H_{k, n+1} \\
& =2 k H_{k, n}-k H_{k, n-1}+2 H_{k, n+2}-H_{k, n+1}
\end{aligned}
$$

as required.

3.3. Theorem. (The $n^{t h}$ Power of the Generating Matrix.) For $n \in \mathbb{N}$, we have

$$
H^{n}=(3+4 k)^{-1}\left[\begin{array}{cc}
2 H_{k, n+2}-H_{k, n+1} & k\left(2 H_{k, n+1}-H_{k, n}\right) \\
2 H_{k, n+1}-H_{k, n} & k\left(2 H_{k, n}-H_{k, n-1}\right)
\end{array}\right]
$$

Proof. Here we shall use induction on $n$. Indeed 3.6 is true for $n=1$. Now we suppose that the $(3.6)$ is true for $n$. Let us show that the result is true for $n+1$, then

$$
\begin{aligned}
& H^{n+1}=(3+4 k)^{-1} {\left[\begin{array}{cc}
2 H_{k, n+2}-H_{k, n+1} & k\left(2 H_{k, n+1}-H_{k, n}\right) \\
2 H_{k, n+1}-H_{k, n} & k\left(2 H_{k, n}-H_{k, n-1}\right)
\end{array}\right]\left[\begin{array}{ll}
2 & k \\
1 & 0
\end{array}\right] } \\
&=(3+4 k)^{-1}\left[\begin{array}{cc}
2\left(2 H_{k, n+2}-H_{k, n+1}\right) & k\left(2 H_{k, n+2}-H_{k, n+1}\right) \\
+k\left(2 H_{k, n+1}-H_{k, n}\right) & \\
2\left(2 H_{k, n+1}-H_{k, n}\right) & k\left(2 H_{k, n+1}-H_{k, n}\right) \\
+k\left(2 H_{k, n}-H_{k, n-1}\right) &
\end{array}\right] \\
&=(3+4 k)^{-1}\left[\begin{array}{cc}
4 H_{k, n+2}-2 H_{k, n+1} & k\left(2 H_{k, n+2}-H_{k, n+1}\right) \\
+2 k H_{k, n+1}-k H_{k, n} & \\
4 H_{k, n+1}-2 H_{k, n} & k\left(2 H_{k, n+1}-H_{k, n}\right) \\
+2 k H_{k, n}-k H_{k, n-1} &
\end{array}\right] \\
&=(3+4 k)^{-1}\left[\begin{array}{cc}
2\left(2 H_{k, n+2}+k H_{k, n+1}\right) & k\left(2 H_{k, n+2}-H_{k, n+1}\right) \\
-\left(2 H_{k, n+1}+k H_{k, n}\right) & \\
2\left(2 H_{k, n+1}+k H_{k, n}\right) & k\left(2 H_{k, n+1}-H_{k, n}\right) \\
-\left(2 H_{k, n}+k H_{k, n-1}\right)
\end{array}\right] \\
&=(3+4 k)^{-1}\left[\begin{array}{ll}
2 H_{k, n+3}-H_{k, n+2} & k\left(2 H_{k, n+2}-H_{k, n+1}\right) \\
2 H_{k, n+2}-H_{k, n+1} & k\left(2 H_{k, n+1}-H_{k, n}\right)
\end{array}\right]
\end{aligned}
$$

as required.

\subsection{Theorem. (Cassini's Identity.) For $n \in \mathbb{N}$}

$$
H_{k, n+1} H_{k, n-1}-H_{k, n}^{2}=(-k)^{n-1}(3+4 k)
$$

Proof. From equation (2.5), $\operatorname{det}\left(H^{n}\right)=(-k)^{n}$ and now from equation (3.6), we get

$$
\begin{aligned}
H^{n}= & (3+4 k)^{-1}\left[\begin{array}{cc}
2 H_{k, n+2}-H_{k, n+1} & k\left(2 H_{k, n+1}-H_{k, n}\right) \\
2 H_{k, n+1}-H_{k, n} & k\left(2 H_{k, n}-H_{k, n-1}\right)
\end{array}\right] \text { then } \\
\operatorname{det}\left(H^{n}\right)= & k(3+4 k)^{-2}\left[\left(2 H_{k, n}-H_{k, n-1}\right)\left(2 H_{k, n+2}-H_{k, n+1}\right)\right. \\
& \left.\quad-\left(2 H_{k, n+1}-H_{k, n}\right)^{2}\right] \\
= & k(3+4 k)^{-2}\left[4 H_{k, n} H_{k, n+2}-2 H_{k, n} H_{k, n+1}-2 H_{k, n-1} H_{k, n+2}\right. \\
& \left.\quad+H_{k, n-1} H_{k, n+1}-4 H_{k, n+1}^{2}-H_{k, n}^{2}+4 H_{k, n+1} H_{k, n}\right]
\end{aligned}
$$

Put $\quad H_{k, n+2}=2 H_{k, n+1}+k H_{k, n} \quad$ and $\quad H_{k, n+1}=2 H_{k, n}+k H_{k, n-1}$ and then

$$
\begin{aligned}
\operatorname{det}\left(H^{n}\right)=k(3+4 k)^{-2}\left[8 H_{k, n} H_{k, n+1}+4 k H_{k, n}^{2}-2 H_{k, n} H_{k, n+1}\right. \\
-4 H_{k, n-1} H_{k, n+1}-2 k H_{k, n-1} H_{k, n}+H_{k, n-1} H_{k, n+1}-16
\end{aligned}
$$


642

$$
\begin{aligned}
& \left.H_{k, n}^{2}-4 k^{2} H_{k, n-1}^{2}-16 k H_{k, n} H_{k, n-1}-H_{k, n}^{2}+4 H_{k, n+1} H_{k, n}\right] \\
= & k(3+4 k)^{-2}\left[4 k H_{k, n}^{2}-17 H_{k, n}^{2}-3 H_{k, n-1} H_{k, n+1}+10 H_{k, n}\right. \\
& \left.H_{k, n+1}-18 H_{k, n} H_{k, n-1}-4 k^{2} H_{k, n-1}^{2}\right] \\
= & k(3+4 k)^{-2}\left[4 k H_{k, n}^{2}-17 H_{k, n}^{2}-3 H_{k, n-1} H_{k, n+1}\right. \\
& \left.+10 H_{k, n}\left(2 H_{k, n}+k H_{k, n-1}\right)-18 H_{k, n} H_{k, n-1}-4 k^{2} H_{k, n-1}^{2}\right] \\
= & k(3+4 k)^{-2}\left[4 k H_{k, n}^{2}-17 H_{k, n}^{2}-3 H_{k, n-1} H_{k, n+1}+20 H_{k, n}^{2}\right. \\
& \left.\quad-8 k H_{k, n} H_{k, n-1}-4 k^{2} H_{k, n-1}^{2}\right] \\
= & k(3+4 k)^{-2}\left[4 k H_{k, n}^{2}+3 H_{k, n}^{2}-3 H_{k, n-1} H_{k, n+1}-4 k H_{k, n-1}\right. \\
& \left.\left(2 H_{k, n}+k H_{k, n-1}\right)\right] \\
= & k(3+4 k)^{-2}\left[4 k H_{k, n}^{2}+3 H_{k, n}^{2}-3 H_{k, n-1} H_{k, n+1}-\right. \\
& \left.4 k H_{k, n-1} H_{k, n+1}\right] \\
= & k(3+4 k)^{-2}\left[(3+4 k) H_{k, n}^{2}-(3+4 k) H_{k, n-1} H_{k, n+1}\right] \\
= & k(3+4 k)^{-1}\left(H_{k, n}^{2}-H_{k, n-1} H_{k, n+1}\right) \\
=- & k(3+4 k)^{-1}\left(H_{k, n-1} H_{k, n+1}-H_{k, n}^{2}\right)
\end{aligned}
$$

Therefore,

$$
\begin{aligned}
& H_{k, n-1} H_{k, n+1}-H_{k, n}^{2}=-(k)^{-1} \operatorname{det}\left(H^{n}\right)(3+4 k) \\
& H_{k, n-1} H_{k, n+1}-H_{k, n}^{2}=(-k)^{-1} \operatorname{det}\left(H^{n}\right)(3+4 k)
\end{aligned}
$$

Since from equation 2.5, $\operatorname{det}\left(H^{n}\right)=(-k)^{n}$ then

$$
H_{k, n-1} H_{k, n+1}-H_{k, n}^{2}=(-k)^{n-1}(3+4 k)
$$

Hence the result.

From the proof of the above theorem we also conclude that

$$
\begin{array}{r}
\left(2 H_{k, n}-H_{k, n-1}\right)\left(2 H_{k, n+2}-H_{k, n+1}\right)-\left(2 H_{k, n+1}-H_{k, n}\right)^{2} \\
=(3+4 k)\left(H_{k, n}^{2}-H_{k, n-1} H_{k, n+1}\right)
\end{array}
$$

3.5. Theorem. For $n \in \mathbb{N}$, we get

$$
\left[\begin{array}{c}
H_{k, n+1} \\
H_{k, n}
\end{array}\right]=\left[\begin{array}{ll}
2 & k \\
1 & 0
\end{array}\right]\left[\begin{array}{c}
H_{k, n} \\
H_{k, n-1}
\end{array}\right]
$$

Proof. To prove the ongoing result we shall use induction on $n$. Indeed the result is true for $n=1$. Suppose that the result is true for $n$ then

$$
\begin{aligned}
{\left[\begin{array}{c}
H_{k, n+2} \\
H_{k, n+1}
\end{array}\right] } & =\left[\begin{array}{c}
2 H_{k, n+1}+k H_{k, n} \\
H_{k, n+1}
\end{array}\right] \\
& =\left[\begin{array}{ll}
2 & k \\
1 & 0
\end{array}\right]\left[\begin{array}{c}
H_{k, n+1} \\
H_{k, n}
\end{array}\right]
\end{aligned}
$$

Since the result is true for $n$ then

$$
\left[\begin{array}{c}
H_{k, n+2} \\
H_{k, n+1}
\end{array}\right]=\left[\begin{array}{ll}
2 & k \\
1 & 0
\end{array}\right]\left[\begin{array}{ll}
2 & k \\
1 & 0
\end{array}\right]\left[\begin{array}{c}
H_{k, n} \\
H_{k, n-1}
\end{array}\right]
$$




$$
\begin{aligned}
& =\left[\begin{array}{ll}
2 & k \\
1 & 0
\end{array}\right]\left[\begin{array}{c}
2 H_{k, n}+k H_{k, n-1} \\
H_{k, n}
\end{array}\right] \\
& =\left[\begin{array}{ll}
2 & k \\
1 & 0
\end{array}\right]\left[\begin{array}{c}
H_{k, n+1} \\
H_{k, n}
\end{array}\right]
\end{aligned}
$$

as desired.

3.6. Theorem. For $n \geq 0$, we get

$$
\left[\begin{array}{c}
H_{k, n+1} \\
H_{k, n}
\end{array}\right]=H^{n}\left[\begin{array}{c}
H_{k, 1} \\
H_{k, 0}
\end{array}\right]
$$

Proof. To prove the ongoing result we shall use induction on $n$. Indeed the result is true for $n=0$. Suppose that the result is true for $n$ then

$$
\left[\begin{array}{ll}
2 & k \\
1 & 0
\end{array}\right]^{n+1}\left[\begin{array}{l}
H_{k, 1} \\
H_{k, 0}
\end{array}\right]=\left[\begin{array}{ll}
2 & k \\
1 & 0
\end{array}\right]\left[\begin{array}{ll}
2 & k \\
1 & 0
\end{array}\right]^{n}\left[\begin{array}{l}
H_{k, 1} \\
H_{k, 0}
\end{array}\right]
$$

Since the result is true for $n$ then

$$
\begin{aligned}
{\left[\begin{array}{ll}
2 & k \\
1 & 0
\end{array}\right]^{n+1}\left[\begin{array}{l}
H_{k, 1} \\
H_{k, 0}
\end{array}\right] } & =\left[\begin{array}{ll}
2 & k \\
1 & 0
\end{array}\right]\left[\begin{array}{c}
H_{k, n+1} \\
H_{k, n}
\end{array}\right] \\
& =\left[\begin{array}{c}
2 H_{k, n+1}+k H_{k, n} \\
H_{k, n+1}
\end{array}\right] \\
& =\left[\begin{array}{c}
H_{k, n+2} \\
H_{k, n+1}
\end{array}\right]
\end{aligned}
$$

as desired.

\section{Binet's Formula by Matrix Diagonalization of Generating Ma- trix}

In this section we will use the diagonalization of the generating matrix 2.5 to obtain Binet's formula for the generalized Fibonacci sequence 2.3 . For this purpose we should prove the following theorem:

4.1. Theorem. For $n \geq 0$

$$
H_{k, n}=\frac{1}{a-b}\left[2\left(a^{n+1}-b^{n+1}\right)-3\left(a^{n}-b^{n}\right)\right]
$$

Proof. The generating matrix is given by $H=\left[\begin{array}{ll}2 & k \\ 1 & 0\end{array}\right]$. Now here our motive is to diagonalize the generating matrix $H$. Since $H$ is a square matrix and so let $\mathrm{x}$ be the eigen value of $H$ and then by the Cayley Hamilton theorem on matrices, we get

$$
\begin{aligned}
|H-x I| & =0 \\
\left|\begin{array}{cc}
2-x & k \\
1 & -x
\end{array}\right| & =0 \\
x^{2}-2 x-k & =0
\end{aligned}
$$

This is the characteristic equation of the generating matrix. Let $a$ and $b$ be the roots of the characteristic equation and also $a$ and $b$ be the two eigen values of the square matrix 
$H$. Now we will try to find the eigen vectors corresponding to the eigen values $a$ and $b$. To find the eigen vectors we simply solve the system of linear equations given by

$$
(H-x I) V=0
$$

where $V$ is the column vector of order $2 \times 1$. First of all we calculate the eigen vector corresponding to the eigen value $a$ and then

$$
\begin{aligned}
(H-a I) V & =0 \\
{\left[\begin{array}{cc}
2-a & k \\
1 & -a
\end{array}\right]\left[\begin{array}{l}
V_{1} \\
V_{2}
\end{array}\right] } & =0 \\
{\left[\begin{array}{c}
2 V_{1}-a V_{1}+V_{2} k \\
V_{1}-a V_{2}
\end{array}\right] } & =0
\end{aligned}
$$

consider the system

$$
\begin{aligned}
(2-a) V_{1}+k V_{2} & =0 \\
V_{1}-a V_{2} & =0
\end{aligned}
$$

and if we take $V_{2}=t$ in equation 4.2 we get $V_{1}=a t$. Hence the eigen vectors corresponding to $a$ are of type $\left[\begin{array}{c}a t \\ t\end{array}\right]$. In particular $t=1$, the eigen vector corresponding to $a$ is $\left[\begin{array}{l}a \\ 1\end{array}\right]$. Similarly the eigen vector corresponding to $b$ is $\left[\begin{array}{l}b \\ 1\end{array}\right]$. Let $A$ be the matrix of eigen vectors, so $A=\left[\begin{array}{ll}a & b \\ 1 & 1\end{array}\right]$ and then

$A^{-1}=(a-b)^{-1}\left[\begin{array}{cc}1 & -b \\ -1 & a\end{array}\right]$. Now we keep the diagonal matrix $D$ in which eigen values of $H$ are on the main diagonal, $D=\left[\begin{array}{ll}a & 0 \\ 0 & b\end{array}\right]$ and then by the diagonalization of matrices, we get

$$
\begin{aligned}
H & =A D A^{-1} \\
H^{n} & =\left(A D A^{-1}\right)^{n} \\
& =A D^{n} A^{-1} \\
& =(a-b)^{-1}\left[\begin{array}{ll}
a & b \\
1 & 1
\end{array}\right]\left[\begin{array}{rr}
a^{n} & 0 \\
0 & b^{n}
\end{array}\right]\left[\begin{array}{cc}
1 & -b \\
-1 & a
\end{array}\right] \\
& =(a-b)^{-1}\left[\begin{array}{cc}
a^{n+1} & b^{n+1} \\
a^{n} & b^{n}
\end{array}\right]\left[\begin{array}{cc}
1 & -b \\
-1 & a
\end{array}\right] \\
& =(a-b)^{-1}\left[\begin{array}{cc}
a^{n+1}-b^{n+1} & -b a^{n+1}+a b^{n+1} \\
a^{n}-b^{n} & -b a^{n}+a b^{n}
\end{array}\right]
\end{aligned}
$$

By using equation 3.10 , we have

$$
\begin{aligned}
{\left[\begin{array}{c}
H_{k, n+1} \\
H_{k, n}
\end{array}\right] } & =(a-b)^{-1}\left[\begin{array}{cc}
a^{n+1}-b^{n+1} & -b a^{n+1}+a b^{n+1} \\
a^{n}-b^{n} & -b a^{n}+a b^{n}
\end{array}\right]\left[\begin{array}{l}
1 \\
2
\end{array}\right] \\
& =(a-b)^{-1}\left[\begin{array}{c}
a^{n+1}-b^{n+1}-2 b a^{n+1}+2 a b^{n+1} \\
a^{n}-b^{n}-2 b a^{n}+2 a b^{n}
\end{array}\right]
\end{aligned}
$$


Let $C=a^{n+1}-b^{n+1}-2 b a^{n+1}+2 a b^{n+1}$ and using $a+b=2$, we achieve

$$
\begin{aligned}
{\left[\begin{array}{c}
H_{k, n+1} \\
H_{k, n}
\end{array}\right] } & =(a-b)^{-1}\left[\begin{array}{c}
C \\
a^{n}-b^{n}-4 a^{n}+2 a^{n+1}+4 b^{n}-2 b^{n+1}
\end{array}\right] \\
& =(a-b)^{-1}\left[\begin{array}{c}
C \\
-3 a^{n}+3 b^{n}+2 a^{n+1}-2 b^{n+1}
\end{array}\right] \\
& =(a-b)^{-1}\left[\begin{array}{c}
C \\
2\left(a^{n+1}-b^{n+1}\right)-3\left(a^{n}-b^{n}\right)
\end{array}\right]
\end{aligned}
$$

Therefore, by equating corresponding terms on both sides we get,

$$
H_{k, n}=\frac{1}{a-b}\left[2\left(a^{n+1}-b^{n+1}\right)-3\left(a^{n}-b^{n}\right)\right]
$$

Hence the proof.

4.2. Theorem. The characteristic roots of $H^{n}$ are

$$
\begin{aligned}
& a^{n}=\frac{Q_{k, n}+\sqrt{Q_{k, n}^{2}-4(-k)^{n}}}{2} \\
& b^{n}=\frac{Q_{k, n}-\sqrt{Q_{k, n}^{2}-4(-k)^{n}}}{2}
\end{aligned}
$$

where $Q_{k . n}$ is the k-Pell-Lucas sequence

Proof. If we write the characteristic polynomial of $H^{n}$, we achieve

$$
\begin{aligned}
& \left|H^{n}-y I\right|=\left|\begin{array}{cc}
\frac{\left(2 H_{k, n+2}-H_{k, n+1}\right)}{3+4 k}-y & k \frac{\left(2 H_{k, n+1}-H_{k, n}\right)}{3+4 k} \\
\frac{\left(2 H_{k, n+1}-H_{k, n}\right)}{3+4 k} & k \frac{\left(2 H_{k, n}-H_{k, n-1}\right)}{3+4 k}-y
\end{array}\right| \\
& =(3+4 k)^{-2}\left|\begin{array}{cc}
\left(2 H_{k, n+2}-H_{k, n+1}\right) & k\left(2 H_{k, n+1}-H_{k, n}\right) \\
-(3+4 k) y & \\
2 H_{k, n+1}-H_{k, n} & k\left(2 H_{k, n}-H_{k, n-1}\right) \\
& -(3+4 k) y
\end{array}\right| \\
& =(3+4 k)^{-2}\left\{\left[\left(2 H_{k, n+2}-H_{k, n+1}\right)-(3+4 k) y\right]\right. \\
& \left.\left[k\left(2 H_{k, n}-H_{k, n-1}\right)-(3+4 k) y\right]-k\left(2 H_{k, n+1}-H_{k, n}\right)^{2}\right\} \\
& =(3+4 k)^{-2}\left[\left(2 H_{k, n+2}-H_{k, n+1}\right) k\left(2 H_{k, n}-H_{k, n-1}\right)\right. \\
& -\left(2 H_{k, n+2}-H_{k, n+1}\right) y(3+4 k)-k y(3+4 k) \\
& \left.\left(2 H_{k, n}-H_{k, n-1}\right)+y^{2}(3+4 k)^{2}-k\left(2 H_{k, n+1}-H_{k, n}\right)^{2}\right] \\
& =(3+4 k)^{-2}\left\{y^{2}(3+4 k)^{2}-y(3+4 k)\left(2 H_{k, n+2}-H_{k, n+1}\right.\right. \\
& \left.+2 k H_{k, n}-k H_{k, n-1}\right)-k\left[\left(2 H_{k, n}-H_{k, n-1}\right)\right. \\
& \left.\left.\left(2 H_{k, n+2}-H_{k, n+1}\right)-k\left(2 H_{k, n+1}-H_{k, n}\right)^{2}\right]\right\} \\
& =(3+4 k)^{-2}\left\{y^{2}(3+4 k)^{2}-y(3+4 k)\left(2 k H_{k, n}-k H_{k, n-1}\right.\right. \\
& \left.+2 H_{k, n+2}-H_{k, n+1}\right)-k\left[\left(2 H_{k, n}-H_{k, n-1}\right)\right. \\
& \left.\left.\left(2 H_{k, n+2}-H_{k, n+1}\right)-k\left(2 H_{k, n+1}-H_{k, n}\right)^{2}\right]\right\}
\end{aligned}
$$


After using equations 3.5, 3.7 and 3.8, we conclude that

$$
\begin{aligned}
\left|H^{n}-y I\right|= & (3+4 k)^{-2}\left[y^{2}(3+4 k)^{2}-y(3+4 k)(3+4 k) Q_{k, n}+k(3+4 k)\right. \\
& \left.\left(H_{k, n}^{2}-H_{k, n-1} H_{k, n+1}\right)\right] \\
= & (3+4 k)^{-2}\left[y^{2}(3+4 k)^{2}-(3+4 k)^{2} Q_{k, n} y+(-k)(3+4 k)\right. \\
& \left.\left(H_{k, n-1} H_{k, n+1}-H_{k, n}^{2}\right)\right] \\
= & (3+4 k)^{-2}\left[y^{2}(3+4 k)^{2}-(3+4 k)^{2} Q_{k, n} y+(-k)(-k)^{n-1}\right. \\
& \left.(3+4 k)^{2}\right] \\
= & (3+4 k)^{-2}\left[y^{2}(3+4 k)^{2}-(3+4 k)^{2} Q_{k, n} y+(-k)^{n}(3+4 k)^{2}\right] \\
= & y^{2}-Q_{k, n} y+(-k)^{n}
\end{aligned}
$$

Hence the characteristic equation of $H^{n}$ is given by

$$
y^{2}-Q_{k, n} y+(-k)^{n}=0
$$

and the characteristic roots are obtained from

$$
y=\frac{Q_{k, n} \pm \sqrt{Q_{k, n}^{2}-4(-k)^{n}}}{2}
$$

Clearly the equation 4.5 has two roots given $a^{n}$ and $b^{n}$ and consequently we get the desired result as

$$
a^{n}=\frac{Q_{k, n}+\sqrt{Q_{k, n}^{2}-4(-k)^{n}}}{2} \text { and } b^{n}=\frac{Q_{k, n}-\sqrt{Q_{k, n}^{2}-4(-k)^{n}}}{2}
$$

Hence the result

4.3. Theorem. The characteristic equation of $H$ is

$$
a^{2}-2 a-k=0
$$

Proof. Here we employ the method of matrices as well as determinants to obtain the characteristic equation for $H$.

Since

$$
\begin{aligned}
& H^{n}=(3+4 k)^{-1} {\left[\begin{array}{cc}
2 H_{k, n+2}-H_{k, n+1} & k\left(2 H_{k, n+1}-H_{k, n}\right) \\
2 H_{k, n+1}-H_{k, n} & k\left(2 H_{k, n}-H_{k, n-1}\right)
\end{array}\right] } \\
& \frac{H^{n}}{H_{k, n-1}}=(3+4 k)^{-1}\left[\begin{array}{cc}
\frac{2 H_{k, n+2}-H_{k, n+1}}{H_{k, n-1}} & \frac{k\left(2 H_{k, n+1}-H_{k, n}\right)}{H_{k, n-1}} \\
\frac{2 H_{k, n+1}-H_{k, n}}{H_{k, n-1}} & \frac{k\left(2 H_{k, n}-H_{k, n-1}\right)}{H_{k, n-1}}
\end{array}\right]
\end{aligned}
$$

Since the ratio of two consecutive generalized Fibonacci numbers is equal to $a$ then

$$
\begin{aligned}
\lim _{n \rightarrow \infty} \frac{2 H_{k, n+2}-H_{k, n+1}}{H_{k, n-1}}= & 2 \lim _{n \rightarrow \infty} \frac{H_{k, n+2}}{H_{k, n-1}}-\lim _{n \rightarrow \infty} \frac{H_{k, n+1}}{H_{k, n-1}} \\
= & 2 \lim _{n \rightarrow \infty} \frac{H_{k, n+2}}{H_{k, n+1}} \lim _{n \rightarrow \infty} \frac{H_{k, n+1}}{H_{k, n}} \lim _{n \rightarrow \infty} \frac{H_{k, n}}{H_{k, n-1}} \\
& \quad-\lim _{n \rightarrow \infty} \frac{H_{k, n+1}}{H_{k, n}} \lim _{n \rightarrow \infty} \frac{H_{k, n}}{H_{k, n-1}} \\
= & 2 a^{3}-a^{2}
\end{aligned}
$$


and

$$
\begin{aligned}
\lim _{n \rightarrow \infty} \frac{2 H_{k, n+1}-H_{k, n}}{H_{k, n-1}} & =2 \lim _{n \rightarrow \infty} \frac{H_{k, n+1}}{H_{k, n-1}}-\lim _{n \rightarrow \infty} \frac{H_{k, n}}{H_{k, n-1}} \\
& =2 \lim _{n \rightarrow \infty} \frac{H_{k, n+1}}{H_{k, n}} \lim _{n \rightarrow \infty} \frac{H_{k, n}}{H_{k, n-1}}-\lim _{n \rightarrow \infty} \frac{H_{k, n}}{H_{k, n-1}} \\
& =2 a^{2}-a
\end{aligned}
$$

Again

$$
\begin{aligned}
\lim _{n \rightarrow \infty} \frac{2 H_{k, n}-H_{k, n-1}}{H_{k, n-1}} & =2 \lim _{n \rightarrow \infty} \frac{H_{k, n}}{H_{k, n-1}}-1 \\
& =2 a-1
\end{aligned}
$$

Therefore,

$$
\lim _{n \rightarrow \infty} \frac{H^{n}}{H_{k, n-1}}=(3+4 k)^{-1}\left[\begin{array}{cc}
(2 a-1) a^{2} & k a(2 a-1) \\
(2 a-1) a & k(2 a-1)
\end{array}\right]
$$

If we consider equation 2.4, we have

$$
\begin{aligned}
& (3+4 k)^{-1}\left[\begin{array}{cc}
(2 a-1) a^{2} & k a(2 a-1) \\
(2 a-1) a & k(2 a-1)
\end{array}\right] \\
& =(3+4 k)^{-1}\left[\begin{array}{cc}
(2 a-1)(2 a+k) & k a(2 a-1) \\
(2 a-1) a & k(2 a-1)
\end{array}\right]
\end{aligned}
$$

If we will compute the determinants of both sides, we get the characteristic equation of the matrix $H$ as follows

$$
\begin{aligned}
& 0=(3+4 k)^{-2}\left[(2 a-1)^{2}\left(2 a k+k^{2}\right)-(2 a-1)^{2} k a^{2}\right] \\
& 0=2 a k+k^{2}-k a^{2} \\
& a^{2}-2 a-k=0
\end{aligned}
$$

as required

\section{References}

[1] Borges, A., Catarino, P., Aires, A. P., Vasco, P. and Campos, H., Two-by-Two matrices involving k-Fibonacci and $k$-Lucas sequences, Applied Mathematical Sciences 8(34), 1659$1666,2014$.

[2] Godase, A. D. and Dhakne, M. B., On the properties of k-Fibonacci and k-Lucas numbers, Int. J. Adv. Appl. Math. and Mech 2 (1),100-106, 2014.

[3] Horadam, A. F., Basic properties of a certain generalized sequence of numbers, Fibonacci Quarterly 3 (3), 161-176, 1965.

[4] Demirturk, B., Fibonacci and Lucas Sums by matrix Methods, International Mathematical Forum 5(3), 99-107, 2010.

[5] Koken, F., and Bozkurt, D., On the Jacobsthal numbers by matrix methods, Int. J. Contemp. Math. Sciences 3(13), 605-614, 2008.

[6] Bilgici, G., New generalizations of Fibonacci and Lucas sequences, Applied Mathematical Sciences, 8 (29), 1429-1437, 2014.

[7] Silvester, J. R., Properties by matrix methods, The Mathematical Gazette 63, (425), 188191, 1979.

[8] Catarino, P., A note involving two-by-two matrices of $k$-Pell and $k$-Pell-Lucas sequences, International Mathematical Forum 8, (32), 1561-1568, 2013.

[9] Catarino, P., and Vasco, P., Some basic properties and a two-by-two matrix involving the k-Pell numbers, Int. Journal of Math. Analysis 7, (45), 209-2215, 2013. 
[10] Ugyun, S., and Eldogan, H., k-Jacobsthal and k-Jacobsthal Lucas matris sequences, International Mathematical Forum 11, (3), 145-154, 2016.

[11] Tasyurdu, Y., Cobanoglu, N., and Dilmen, Z., On the a new family of k-Fibonacci numbers, Journal of Science and Technology 9, (1), 95-101, 2016. 